\title{
Breakthrough Listen Observations of 1I/'Oumuamua with the GBT
}

\author{
J. Emilio Enriquez, ${ }^{1,2}$ Andrew Siemion, ${ }^{1,2,3}$ T. Joseph W. Lazio, ${ }^{4}$ Matt Lebofsky ${ }^{1}$ David H. E. MacMahon, ${ }^{1}$ Ryan S. Park, ${ }^{4}$ \\ Steve Croft, ${ }^{1}$ David DeBoer, ${ }^{1}$ Nectaria Gizani, ${ }^{1,5}$ Vishal Gajjar, ${ }^{6}$ Greg Hellbourg, ${ }^{1}$ Howard Isaacson, ${ }^{1}$ and Danny C. Price ${ }^{1,7}$ \\ ${ }^{1}$ Department of Astronomy, University of California, Berkeley \\ ${ }^{2}$ Department of Astrophysics/IMAPP,Radboud University, Nijmegen, Netherlands \\ ${ }^{3}$ SETI Institute, Mountain View, California \\ ${ }^{4}$ Jet Propulsion Laboratory, California Institute of Technology, Pasadena, California \\ ${ }^{5}$ School of Science and Technology, Hellenic Open University, Greece \\ ${ }^{6}$ Space Sciences Laboratory, University of California, Berkeley \\ ${ }^{7}$ Centre for Astrophysics \& Supercomputing, Swinburne University of Technology, Australia
}

Keywords: asteroids - extraterrestrial intelligence

\section{INTRODUCTION.}

On 2017 October 18, the Pan-STARRS collaboration discovered an object within our solar system that appeared to be on a hyperbolic orbit (Williams 2017). Subsequent observations suggest this object (now designated 1I/2017 U1 or $1 \mathrm{I} /$ 'Oumuamua) is of interstellar origin, lacks a coma, and that its shape is highly elongated relative to other known asteroids (Meech et al. 2017).

It has long been suggested that advanced extraterrestrial civilizations, should they exist, could conceivably send probes to other stars either for exploration or communication purposes (Bracewell 1960; Freitas 1980; Rose \& Wright 2004; Gertz 2016). Interstellar probes would likely be equipped with communication technology that could potentially be operating in the radio band.

The Breakthrough Listen (BL) program (Worden et al. 2017) conducted an observing campaign targeting 1I/'Oumuamua with the Robert C. Byrd Green Bank Telescope (GBT), with a goal of detecting, or placing limits on, radio emission consistent with a technological source. Because 1I/'Oumuamua is much closer to Earth than typical stellar targets, we have the opportunity to carry out a search for extremely weak transmitters: unprecedented in any other SETI experiment. Here we present a description of the observing campaign, and preliminary results.

\section{OBSERVATIONS}

In December 2017, the BL Team conducted an initial 8-hour observation campaign using the GBT with four different receivers: L-band (1.1-1.9 GHz), S-band (1.73-2.6 GHz), C-band (4-8 GHz) and X-band (8-11.6 GHz). Observations were conducted using the Breakthrough Listen back-end (MacMahon et al. 2017) to collect and analyze the data.

The 8-hour block was divided into two hours per receiver. Considering the suggested rotation of 1I/'Oumuamua, we subdivided the two hours per receiver into half-hour blocks, one for each phase quadrant of the rotation. Each half hour was further subdivided into six 5-minute observations in an ABACAD configuration as described in Isaacson et al. (2017) and Enriquez et al. (2017).

We set the time of the first observation as $t_{0}$ (2017 December 13 at 21:53:22 UTC; 58100.9121 MJD), and used a rotational period of $8.1 \pm 0.02 \mathrm{hrs}$ from Bolin et al. (2017) for the phase calculation. Over two weeks, we covered the full rotational phase of $1 \mathrm{I} /$ 'Oumuamua with each receiver. Table 1 shows the MJD dates relating receiver band coverage to quadrant phase. 
Table 1. Observation MJD dates for a given quadrant/band combination. The quadrants Q1, Q2, Q3 and Q4 are defined in phase space as $0 .-0.25,0.25-0.5,0.5-0.75$, and $0.75-1.0$ respectively.

\begin{tabular}{ccccc}
\hline & L-band & S-band & C-band & X-band \\
\hline \hline & & & & \\
Q1 & 58110.0323 & 58100.9121 & 58106.0037 & 58100.9791 \\
& & & & \\
Q2 & 58101.0313 & 58106.0570 & 58107.1261 & 58117.9094 \\
& & & & \\
Q3 & 58107.1768 & 58109.9187 & 58101.09403 & 58109.8753 \\
& & & & \\
Q4 & 58109.9994 & 58105.8901 & 58109.9749 & 58105.9473 \\
& & & & \\
\hline
\end{tabular}

\section{RESULTS AND DISCUSSION}

We conducted a search of the data for narrow-band ( $3 \mathrm{~Hz}$ resolution) drifting sinusoids as described by Enriquez et al. (2017), over a drift rate range of $\pm 2 \mathrm{~Hz} / \mathrm{s}$. This range includes any acceleration in the geocentric and barycentric frames, as well as accommodating a transmitter located anywhere on the body itself. Our preliminary results show no narrow band radio emission from the direction of $1 \mathrm{I} /{ }^{\prime}$ Oumuamua at any rotational phase.

The object was at $\sim 2$ AU when observed, and given an approximate SEFD of 20 Jy, with a 300 s observation, and a $5 \sigma$ threshold, these observations were sensitive to a hypothetical transmitter with an EIRP of $\sim 0.08 \mathrm{~W} \quad(\sim 3,000$ times weaker than the Dawn spacecraft communication down-link.).

Based on the possibility that 1I/'Oumuamua could be in fact a dormant comet with delayed outgassing, we also searched for any indication of hydroxyl emission at the four transitions between $1612 \mathrm{MHz}$ and $1720 \mathrm{MHz}$. We searched the L-band data taken during quadrant Q2 by stacking the three 5-min observations. No emission was detected, confirming previous observations during closer approach that the nature of the object is consistent with an asteroidlike composition (Park 2017 in prep).

All data collected by BL will be publicly available. A subset of the observations described here can be downloaded from the Breakthrough Listen Public Web Archive.

Funding for BL is provided by the Breakthrough Prize Foundation. Part of this research was carried out at the Jet Propulsion Laboratory, California Institute of Technology, under a contract with the National Aeronautics and Space Administration.

\section{REFERENCES}

Bolin, B. T., Weaver, H. A., Fernandez, Y. R., et al. 2017, ArXiv e-prints, arXiv:1711.04927

Bracewell, R. N. 1960, Nature, 186, 670

Enriquez, J. E., Siemion, A., Foster, G., et al. 2017, ApJ, 849, 104

Freitas, R. A. J. 1980, British Interplanetary Society, 33, 95

Gertz, J. 2016, arXiv.org, 1609.04635v1

Isaacson, H., Siemion, A. P. V., Marcy, G. W., et al. 2017, PASP, 129, 054501

MacMahon, D., et al. 2017, PASP; in Press

Meech, K. J., Weryk, R., Micheli, M., et al. 2017, Nature, $552,378 \mathrm{EP}$
Park, R., Pisano, D., Lazio, T., Chodas, P., \& Naidu, S. 2017, submitted to AJ

Rose, C., \& Wright, G. 2004, Nature, 431, 47

Williams, G. 2017, MPEC 2017-U181: COMET C/2017 U1

(PANSTARRS), Minor Planet Electronic Circular, MPEC

Worden, S. P., Drew, J., Siemion, A., et al. 2017, Acta Astronautica, 139, 98 